\title{
Anaesthesia for paediatric patients: Minimising the risk
}

\author{
K Bester, MB ChB, DA (SA), FCA (SA); H Meyer, MB ChB, FRCA; M Crowther, MB ChB, DA (SA), Dip Obst (SA); \\ R Gray, MB ChB, DA (SA), FCA (SA) \\ Division of Paediatric Anaesthesia, Department of Anaesthesia and Perioperative Medicine, Faculty of Health Sciences, \\ University of Cape Town, South Africa
}

Corresponding author: K Bester (kotgel@gmail.com)

\begin{abstract}
Advances in paediatric anaesthesia include the availability of dedicated facilities with appropriate equipment, monitoring and specialised personnel trained in paediatric anaesthesia and resuscitation. These developments have contributed to the reduction in perioperative risk for paediatric patients. However, access to all the resources available in dedicated paediatric facilities is limited in resource-constrained settings. The objective of this review is to provide guidance with regard to the selection of suitable facilities for perioperative care, risk stratification and patient selection, safe selection of medication and standardisation of its use, and implementation of specific anaesthetic techniques that can minimise risk in the paediatric surgical population.
\end{abstract}

S Afr Med J 2018;108(6):457-459. DOI:10.7196/SAMJ.2018.v108i6.13351

On 28 January 1848, 15-year-old Hannah Greener died after receiving a chloroform anaesthetic for the removal of a toenail. This was the first recorded death attributed to anaesthesia. Subsequent advances in anaesthetic techniques, training, medication and monitoring have contributed significantly to a decline in anaesthesia-related mortality and morbidity. ${ }^{[1,2]}$ The development of dedicated paediatric centres and paediatric specialties has also led to the improvement in outcomes seen in paediatric surgery. ${ }^{[3]}$

The American Academy of Pediatrics Guidelines for the Pediatric Perioperative Anesthesia Environment suggest that a number of requirements should be met to minimise perioperative risk. These include staff, such as anaesthetists, nursing and technical personnel with dedicated paediatric training, and a treatment area with appropriate equipment and medication. ${ }^{[4]}$

In resource-limited settings, the risks of providing surgical services to paediatric patients need to be offset against the need for optimal utilisation of national resources available for the provision of services. When considering the development of paediatric surgical services, the following principles can be applied to help to minimise risk in this patient population:

- Facilities should be appropriately equipped to manage paediatric patients and the complications that may arise from the procedure(s) undertaken.

- Risk stratification of paediatric patients for specific procedures in specific settings should be undertaken.

- Standardised operating procedures and drug-dosing tables should be used to promote simplicity and safety.

- Techniques employed by the anaesthetist should be chosen to minimise risk, and consideration must be given to appropriate postoperative analgesia and monitoring requirements.

\section{Facillities}

When planning services, a facility should aim to manage a minimum number of specified cases, ensure appropriate skills, and maintain equipment and drugs for their spectrum of practice - preferably written into policy. Clear recommendations with regard to these minimum numbers remain controversial, but complication rates have been reported to be significantly increased in groups performing
$<100$ paediatric anaesthesia procedures annually than in groups performing $>200$ procedures. ${ }^{[5]}$ In 2006 , a major review of services for children in hospitals in the UK recommended that anaesthetists looking after children should maintain an annual caseload of $\geq 100$ patients between the ages of 0 and 12 years. $^{[6]}$

All equipment should be the appropriate size and type for paediatric patients. Many facilities set up a 'paediatric trolley', which should have a list of the required equipment that should be checked regularly. Pre- and postoperative care facilities should also fulfil certain requirements. The recovery area should have one-onone patient-to-nurse ratios and the staff should be familiar with specific paediatric care and resuscitation protocols. Depending on the complexity of cases, the need for availability of overnight, high-care or intensive care facilities should be addressed.

Many children in South Africa (SA) require surgery in small hospitals, and the challenge is to improve the structures and processes of care in those hospitals. The role of tertiary hospitals should be to support colleagues in smaller district hospitals by means of telephonic advice, the sharing of protocols and educational opportunities, and reviewing digitally transmitted images where possible.

\section{Risk stratification}

Each facility should decide on their scope of practice based on their ability to deal with potential risks. The caseload will need to be estimated as accurately as possible and the type and complexity of surgery should be considered (Table 1 ). ${ }^{[7,8]}$

Consideration should be given to the possibility of localising children's surgery to one location or within a group of small/collegiate hospitals to achieve a sufficient workload to maintain competence.

\section{Age}

The neonatal period is associated with highest risk, and neonatal surgery should only be undertaken in specialist centres. Children $<1$ year of age undergoing surgery have been shown to have a 4.5 times increased risk of perioperative mortality compared with older children. ${ }^{[9]}$ Risk of perioperative adverse events decreases with age, and the likelihood of having an adverse event in the perioperative period decreases by $3 \%$ for every 1 -year increase in age. ${ }^{[10]}$ 
Table 1. Recommendations for paediatric surgery (British Association of Paediatric Surgeons)

\begin{tabular}{ll}
\hline Type of hospital & Surgical procedure \\
\hline $\begin{array}{l}\text { Non-specialist paediatric } \\
\text { surgery safe to be performed at } \\
\text { peripheral hospital }{ }^{[7]}\end{array}$ & Elective \\
& Congenital inguinal hernia \\
& Congenital hydrocele \\
& Circumcision \\
& Orchidopexy \\
& Umbilical hernia repair \\
& Emergency \\
& Appendicectomy \\
& Correction of torsion of testis \\
& Repair of incarcerated hernia \\
& Less complex trauma \\
Specialist paediatric surgery & Neonatal surgery \\
requiring specialist paediatric & Complex surgical conditions \\
centre $^{[8]}$ & requiring special expertise \\
& Children with significant \\
& comorbidities \\
& Paediatric urology
\end{tabular}

\section{Planning for the true emergency}

The need for urgent surgery has been shown to be a better predictor of risk than the type of surgery, ${ }^{[1]}$ with emergent or urgent cases accounting for $83 \%$ of in-hospital perioperative deaths ${ }^{[9]}$ and $88 \%$ of unplanned intensive care admissions. ${ }^{[12]}$ While it may not be in the patients' interests to defer emergent surgery and transfer them to an alternative facility, it may be preferable if the outcome is likely to be better in a centre where the expertise and resources are available. The decision to operate is based on the child's age, availability of a competent team, geographical location or response to initial treatment. Examples of true emergencies, where it may be better for a relatively inexperienced team to operate immediately, include torsion of the testis, volvulus, expanding intracranial haemorrhage, fracture with neurovascular compromise or upper airway obstruction.

\section{Comorbidities}

Significant pathological conditions, such as the presence of a syndrome, a large burn injury or a cardiac lesion, are usually clearly evident. However, there are several comorbidities that may have a significant impact on the perioperative course, which may only become evident when actively sought. These include the irritable airway, presence of obstructive sleep apnoea (OSA), neuromuscular disease and a history of premature birth.

Airways may be irritable in patients with a history of upper respiratory tract infection (URTI) during the preceding 2 weeks, or a personal or family history of asthma or atopy. These patients have a higher incidence of laryngospasm, bronchospasm, stridor, desaturation and other airway complications. On average, very young children will have 6 - 8 URTIs every year. While it may be possible to delay elective surgery, the decision to proceed must be balanced against the urgency of the procedure and potential risk for the patient.

OSA and obesity are associated with an increased risk of respiratory depression and adverse airway events. ${ }^{[10]}$ OSA may also be complicated by pulmonary hypertension and cor pulmonale. Patients with severe OSA are not suitable for day surgery, require reduced opioid doses and postoperative apnoea and oxygen saturation monitoring for 24 - 48 hours.

Neuromuscular disease can complicate the perioperative course owing to involvement of the cardiovascular, respiratory and gastrointestinal systems. Patients may also be at risk of adverse reactions to anaesthetic drugs, such as anaesthesia-induced rhabdomyolysis (AIR), malignant hyperthermia ( $\mathrm{MH}$ ) and mitochondrial dysfunction. ${ }^{[13]}$

A history of prematurity should be elicited in all children. Prematurity may have many long-term consequences that will impact on perioperative management. These include chronic lung disease, acquired subglottic stenosis secondary to prolonged intubation, and cerebral palsy. Subglottic stenosis can result in difficulty with intubation and may require the use of a smaller endotracheal tube than anticipated.

\section{Medication and standardisation}

The administration of medication to paediatric patients requires meticulous attention to detail. Use of drug tables, calculators and cross-checking by healthcare workers can lessen potential errors. ${ }^{[4]} \mathrm{A}$ simple chart that lists doses of emergency drugs and recipes for mixture of inotropic infusions can be of great assistance. It is also helpful to display algorithms for the management of emergency situations. Duplication of drug administration between the theatre and ward is a common problem and systems should be in place to avoid such errors.

Halothane causes an increased incidence of bradycardia and myocardial depression in children compared with sevoflurane. Standard vaporisers allow for seven times the effective dose of halothane to be delivered, which explains the high incidence of intraoperative cardiac arrests owing to overdose of inhalational agents reported in the older literature. ${ }^{[1,2]}$ It should be noted that sevoflurane overdose can also result in cardiac arrest, and $8 \%$ sevoflurane should be used with caution.

One should be cognisant of potential side-effects when administering neuromuscular-blocking agents. Muscle relaxation is required less frequently in children, and intubation may often be safely achieved with the use of medications other than muscle relaxants. Suxamethonium may offer superior intubating conditions to the non-depolarising muscle relaxants, but it can cause lifethreatening hyperkalaemia in susceptible patients, and may cause bradycardia. ${ }^{[3]}$ In young children who may suffer from undiagnosed myopathies and muscle dystrophies, the risk of AIR and MH is of concern wherever volatiles or suxamethonium are used. ${ }^{[13]}$ Muscle relaxants should always be reversed in doses titrated to findings on neuromuscular monitoring to reduce the risk of respiratory complications.

The volumes of fluid used during administration of medication may represent a significant proportion of the total volume administered, particularly in younger patients. It is important that the anaesthetist is aware of the volume of dead space in intravenous systems. Every drug should be flushed in with a specified volume, and care should be taken to ensure that the system is completely cleared of medication before the patient leaves theatre. A small volume of medication may correlate to a significant dose of the drug in a small patient.

Practitioners must be vigilant in their assessment of a patient's volume status and blood loss. Underestimation of preoperative or intraoperative blood loss can lead to hypovolaemic cardiac arrest in children. Reports of cardiac arrest due to hypovolaemia were often related to operations where blood loss might have been difficult to estimate or could go unnoticed, e.g. craniectomies and spinal surgery. ${ }^{[2]}$ Where there is a high risk of bleeding, advanced haemodynamic monitoring should be employed, e.g. invasive blood pressure monitoring, echocardiography and near-infrared spectroscopy. It is important to recognise that the use of advanced monitoring should not delay the ongoing resuscitation in these patients. 


\section{Techniques \\ Airway management}

Respiratory complications in children are more common than cardiac complications, and younger children are more vulnerable to such events. ${ }^{[1]}$ Respiratory episodes are frequently responsible for unexpected intensive care unit admissions and cardiac arrest. ${ }^{[2,12]}$

A history of URTI or atopy, surgery to the neck and airway, and younger age $(<6$ years) increases the risk of adverse respiratory episodes. ${ }^{[2,10,14]}$ The most appropriate technique should be chosen and the anaesthetist must be prepared to manage such potential adverse events. Adverse airway episodes are not limited to the operating room. It has been shown that $\sim 50 \%$ of all cardiac arrests in the recovery area are caused by adverse respiratory events. ${ }^{[14]}$

The type of airway device used can impact on the risk of adverse respiratory events. The use of a face mask only reduces the risk of respiratory complications. The use of a supraglottic device may decrease complications in the recovery room, ${ }^{[14]}$ but it may increase adverse respiratory events intraoperatively, especially in children weighing $<12 \mathrm{~kg}$. Complications experienced with supraglottic devices tended to be more mechanical in nature (difficulty with placement and ventilation, dislodgement), while tracheal tubes more commonly caused laryngospasm and bronchospasm. ${ }^{[15]}$ Cuffed tracheal tubes, especially where the cuff pressure is monitored, are associated with fewer perioperative respiratory complications. ${ }^{[14]}$ Desflurane may increase the risk of adverse respiratory events in children and should be avoided in susceptible patients.

\section{Ultrasound}

Ultrasound may enhance the success rate and decrease complications when used for vascular access and regional techniques. ${ }^{[16]}$ In our experience, it can be an invaluable tool when vascular access is difficult. While guidelines from North America and the UK recommend that ultrasound imaging be used for vascular access in children, ${ }^{[16]}$ it may not be readily available in many hospitals. It therefore remains important that clinicians maintain their skills in both ultrasound-guided and landmark techniques.

\section{Human factors}

Human factors contribute significantly to adverse events. A study showed that insufficient practical application of techniques contributed more to critical events than lack of knowledge. ${ }^{[1]} \mathrm{A}$ retrospective analysis by Marcus ${ }^{[17]}$ highlighted the significant role of poor clinical decision-making, inadequate checking, practical task failures, lack of experience, poor communication and distraction with regard to adverse events.

Although dedicated paediatric anaesthetists and centres lead to better outcomes, these resources are insufficient to accommodate the needs of paediatric patients in SA. Every institution that can contribute towards the care of paediatric patients should therefore evaluate their system and choose to manage an appropriate number of suitable cases, using the correct equipment and medication.
Maintaining paediatric anaesthetic skills may be achieved by refresher courses at larger units and attending advanced paediatric life support courses - an 18-month interval has been suggested.

\section{Conclusion}

Facilities providing surgery to paediatric patients should be able to manage all perioperative aspects of patient care, including potential complications. A culture of critical review and redress will ensure that facilities maintain adequate standards of care. Co-operation, partnerships and networks are indispensable in the pursuit of excellence in paediatric anaesthesia.

\section{Acknowledgements. None.}

Author contributions. KB: substantial contribution to conception, initial and further writing, revision and final approval of work. HM and RG: substantial contribution to further writing, revision and final approval of work. MC: contribution to research for and writing of a section, i.e. Techniques.

Funding. None.

Conflicts of interest. None.

1. Gonzalez LP, Pignaton W, Kusano PS, Módolo NSP, Braz JRC, Braz LG. Anesthesia-related mortality in pediatric patients: A systematic review. Clinics (Sao Paulo) 2012;67(4):381-387. https://dol. org/10.6061/clinics/2012(04)12

2. Morray JP. Cardiac arrest in anesthetized children: Recent advances and challenges for the future Paediatr Anaesth 2011;21(7):722-729. https://doi.org/10.1111/j.1460-9592.2010.03440.x

3. Somri M, Coran AG, Hadjittofi C, et al. Improved outcomes in paediatric anaesthesia: Contributing 3. Somri M, Coran AG, Hadjittofi C, et al. Improved outcomes in paediatric anaesthesia:
factors. Pediatr Surg Int 2012;28(6):553-561. https://doi.org/10.1007/s00383-012-3101-y

factors. Pediatr Surg Int 2012;28(6):553-561. https://doi.org/ $10.1007 / 500383-012-3101-y$
Village EG. Guidelines for the pediatric perioperative anesthesia environment. Pediatrics 1999;103(2):512 515. https://doi.org/10.1542/peds.103.2.512

. Auroy Y, Ecoffey C, Messiah A, Rouvier B. Relationship between complications of pediatric anesthesia and volume of pediatric anesthetics. Anesth Analg 1997;84:228-236. https://doi.org/10.1097/00000539199701000-0006

6. Healthcare Commission. Improving services for children in hospital. 2007. http://www.ehealthnurse org.uk/pdf/ChildrenReportFeb07.pdf (accessed 20 April 2018).

7. British Association of Paediatric Surgeons. A Guide for Purchasers and Providers of Paediatric Surgica Services. Edinburgh: The Royal College of Surgeons of Edinburgh, 1995.

Arul G, Spicer R, McDonald P, Robinson P, Spitz L. Where should paediatric surgery be performed? Arch Dis Child 1998;79(1):65-72. https://doi.org/10.1136/adc.79.1.65

Meyer HM, Thomas J, Wilson GS, de Kock M. Anesthesia-related and perioperative mortality: An audit of 8493 cases at a tertiary pediatric teaching hospital in South Africa. Pediatr Anesth 2017;27(10):1021-1027. https://doi.org/10.1111/pan.13214

10. Habre W, Disma N, Virag K, et al. Incidence of severe critical events in paediatric anaesthesia (APRICOT): A prospective multicentre observational study in 261 hospitals in Europe. Lancet Respir Med (APRICOT): A prospective multicentre observational study in 261 hosp 11. Nasr VG, DiNardo JA, Faraoni D. Development of a pediatric risk assessment score to predict peri-
operative mortality in children undergoing noncardiac surgery. Anesth Analg 2017;124(5):1514-1519. operative mortality in children undergoing nonca

12. Gibson AR, Limb J, Bell G. Retrospective audit of unplanned admissions to pediatric high dependency and intensive care after surgery. Paediatr Anaesth 2014;24(4):372-376. https://doi.org/ $10.1111 /$ pan. 12343

3. Gray RM. Anaesthesia and the paediatric muscle disorders. South Afr J Anaesth Analg 2013;19(1):20-23. https://doi.org/10.1080/22201173.2013.10872885

4. Von Ungern-Sternberg BS. Respiratory complications in the pediatric postanesthesia care unit. Anesthesiol Clin 2014;32(1):45-61. https://doi.org/10.1016/j.anclin.2013.10.004

5. Bordet F, Allaouchiche B, Lansiaux S, et al. Risk factors for airway complications during genera anaesthesia in paediatric patients. Paediatr Anaesth 2002;12(9):762-769. https://doi.org/10.1046/j.14609592.2002.00987.x

16. Kamra K, Hammer GB. Central venous catheter placement in children: 'How good is good enough?' Paediatr Anaesth 2013;23(11):971-973. https://doi.org/10.1111/pan.12228

17. Marcus R. Human factors in pediatric anesthesia incidents. Paediatr Anaesth 2006;16(3):242-245. https://doi.org/10.1111/j.1460-9592.2005.01771.x 